\title{
Clonidine increases bone resorption in humans
}

\author{
E. J. Limonard ${ }^{1}$. T. Schoenmaker ${ }^{2}$ - T. J. de Vries ${ }^{2} \cdot$ M. W. Tanck ${ }^{3}$.

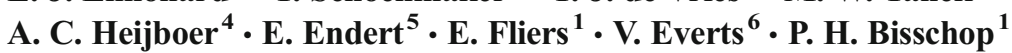

Received: 26 May 2015 / Accepted: 1 September 2015 / Published online: 6 October 2015

(C) The Author(s) 2015. This article is published with open access at Springerlink.com

\begin{abstract}
Summary Inhibition of sympathetic signaling to bone reduces bone resorption in rodents. In contrast, we show that pharmacological reduction of the sympathetic tone increases bone resorption in humans in vivo. This effect does not appear to be mediated via a direct pharmacological effect on the osteoclast.

Introduction Inhibition of sympathetic signaling to bone reduces bone resorption in rodents. It is uncertain whether a similar role for the sympathetic nervous system exists in humans. The sympathetic tone can be reduced by clonidine, which acts via alpha-2-adrenergic receptors in the brainstem. Our objective was to determine the effect of clonidine on bone turnover in humans.
\end{abstract}

\section{P. H. Bisschop}

p.h.bisschop@amc.uva.nl

E. J. Limonard

e.j.limonard@amc.uva.nl

T. Schoenmaker

t.schoenmaker@acta.nl

T. J. de Vries

teun.devries@acta.nl

M. W. Tanck

m.w.tanck@amc.uva.nl

A. C. Heijboer

a.heijboer@vumc.nl

E. Endert

e.endert@amc.uva.nl

E. Fliers

e.fliers@amc.uva.nl

V. Everts

v.everts@acta.nl
Methods The acute effect of a single oral dose of $0.3 \mathrm{mg}$ clonidine on serum bone turnover markers (C-terminal crosslinking telopeptides of collagen type I (CTx), a marker for bone resorption, and procollagen type $1 \mathrm{~N}$ propeptide (P1NP), a marker for bone formation) was determined in a randomized crossover design in 12 healthy volunteers, aged 18-70 years. In addition, we assessed the effect of clonidine on the number of tartrate-resistant acid phosphatase-positive multinucleated cells (TRAcP ${ }^{+} \mathrm{MNCs}$ ) and bone resorption.

Results CTx concentrations increased after clonidine treatment compared to the control condition $(p=0.035)$. P1NP concentrations were not affected by clonidine $(p=0.520)$.

In vitro, clonidine had no effect on the number of TRAcP ${ }^{+}$ MNCs $(p=0.513)$ or on bone resorption $(p=0.996)$.

1 Department of Endocrinology and Metabolism, Academic Medical Center, University of Amsterdam, PO Box 22660, 1100 DD Amsterdam, Netherlands

2 Department of Periodontology, Academic Centre for Dentistry Amsterdam, University of Amsterdam and VU University, MOVE Research Institute, Gustav Mahlerlaan 3004, 1081 LA Amsterdam, Netherlands

3 Department of Clinical Epidemiology, Biostatistics and Bioinformatics, Academic Medical Center, University of Amsterdam, PO Box 22660, 1100 DD Amsterdam, Netherlands

4 Department of Clinical Chemistry, Endocrine Laboratory, VU University Medical Center, PO Box 7057, 1007 MB Amsterdam, Netherlands

5 Department of Clinical Chemistry, Laboratory of Endocrinology, Academic Medical Center, University of Amsterdam, PO Box 22660, 1100 DD Amsterdam, Netherlands

6 Department of Oral Cell Biology, Academic Centre for Dentistry Amsterdam, University of Amsterdam and VU University, MOVE Research Institute, Gustav Mahlerlaan 3004, 1081

LA Amsterdam, Netherlands 
Conclusions We demonstrated that clonidine increases bone resorption in humans in vivo. This effect does not appear to be mediated via a direct effect on the osteoclast.

Keywords Alpha-2-adrenergic receptor · Bone turnover . Osteoclast · Sympathetic nervous system

\section{Introduction}

Bone remodeling is under control of the sympathetic nervous system [1-3]. Knockout of the beta-2-adrenergic receptor $(\beta 2-\mathrm{AR})$ in osteoblasts resulted in a high bone mass phenotype [4]. In line, administration of a $\beta$-blocker or $\beta$-agonist was shown to increase and decrease bone mass in mice, respectively [5-7]. The role of the sympathetic nervous system in bone turnover was further supported by the high bone mass phenotype in mice with low sympathetic activity, due to dopamine $\beta$-hydroxylase deficiency [8]. Recently, the role of the sympathetic nervous system in bone metabolism was extended by investigating mice with a double knockout of alpha- $2_{\mathrm{A}^{-}}$and alpha- $\mathrm{C}_{\mathrm{C}^{-}}$adrenoceptor genes [9]. Alpha-2adrenorecoptors are expressed in the brainstem, and when activated, sympathetic activity is reduced. Consequently, knockout of alpha-2-adrenoceptors resulted in chronic sympathetic hyperactivity, indicated by increased norepinephrine release. Unexpectedly, these mice presented with a phenotype of high bone mass with increased bone formation and decreased bone resorption.

It is uncertain whether human bone metabolism is also under control of the sympathetic nervous system. Epidemiological studies on the association between betablocker use and fracture risk showed inconclusive results, with $\beta$-blockers having positive, negative, or no effects on bone mass [10-22]. Two recent meta-analyses indicate a small but significant risk reduction (15 and $17 \%$, respectively) of any fracture in patients treated with beta-blockers [23, 24]. This risk reduction was, however, associated with the use of beta-1-selective blockers, rather than non-selective betablockers. A case-control study comparing bone turnover in pheochromocytoma patients and controls showed that pheochromocytoma patients have increased bone resorption, which normalizes after adrenalectomy $[25,26]$. These findings support the concept of regulation of bone remodeling by the sympathetic nervous system in humans.

Pharmacological activation of alpha-2-adrenergic receptors in the brainstem by clonidine leads to a reduction in sympathetic tone. Clonidine is typically prescribed to treat hypertension and menopausal flushes. To study the effect of the sympathetic nervous system on bone in humans, we conducted a crossover study determining the acute effect of a single oral dose of $0.3 \mathrm{mg}$ clonidine (a selective alpha-2-adrenergic receptor agonist) on bone turnover markers in 12 healthy subjects. We hypothesized that pharmacological alpha-2adrenergic stimulation decreases $\mathrm{C}$-terminal cross-linking telopeptides of collagen type I (CTx) and procollagen type $1 \mathrm{~N}$ propeptide (P1NP) concentrations, indicating decreased bone turnover. Unexpectedly, CTx concentrations increased after clonidine treatment compared to the control condition, indicating enhanced bone resorption. In view of these results, we further investigated whether the effects of clonidine are mediated via direct stimulation of alpha-2-adrenergic receptors on the osteoclast by determining the effect of clonidine on osteoclastogenesis and bone resorption in vitro.

\section{Subjects and Methods}

\section{In vivo effect of clonidine on bone formation and resorption}

\section{Subjects}

Twelve healthy female and male volunteers aged $18-70$ years were recruited for this crossover study. Exclusion criteria for participation were (1) contra-indications to clonidine, i.e., hypersensitivity, pulse rate $<50$ per minute, and use of antihypertensive drugs or drugs with negative chronotropic effects on the heart; (2) any medication or disease influencing bone turnover; (3) blood pressure $<110 / 70 \mathrm{mmHg}$; and (4) pregnancy. At baseline, we took a complete history and measured body weight, height, and blood pressure. A venous blood sample was drawn after an overnight fast to determine serum concentrations of calcium, albumin, phosphate, parathyroid hormone $(\mathrm{PTH}), 25$-hydroxyvitamin D $(25(\mathrm{OH}) \mathrm{D})$, creatinine, and alkaline phosphatase.

\section{Study design}

This open-label crossover trial was performed at the Department of Endocrinology and Metabolism of the Academic Medical Center of the University of Amsterdam (AMC/UvA) in the Netherlands. All participating subjects were studied two times (clonidine and control) and served as their own control. The 2 study days were separated by a washout period of at least 1 week. Participants were randomly allocated to clonidine followed by no intervention or no intervention followed by clonidine using a computer-generated randomization list (nQuery Advisor version 7.0, Statistical Solutions, Cork, Ireland). An antecubital vein of the arm was cannulated for all blood sampling. A baseline blood sample was drawn between 8:30 and 9:00 h to determine bone turnover markers (C-terminal cross-linking telopeptides of collagen type I (CTx) and procollagen type $1 \mathrm{~N}$ propeptide (P1NP)), normetanephrine, and creatinine concentrations in plasma or serum, after which participants received either a 
single oral dose of clonidine (a selective alpha-2-adrenoceptor agonist) $0.3 \mathrm{mg}$ or no intervention at 9:00 h. Five blood samples were drawn during the following $6 \mathrm{~h}$ to determine bone turnover markers, normetanephrine, and creatinine concentrations. All blood samples were centrifuged within $30 \mathrm{~min}$ and stored at $-20^{\circ} \mathrm{C}$ until analysis. Participants were fasted throughout the experiment but were allowed to drink water. They were restricted to bed rest with minimal physical activity. During the experiment, blood pressure and heart rate were monitored every 15 min, with a Microlife ${ }^{\circledR}$ BP 3 AC1-1 automatic device. The study was approved by the Institutional Review Board of the Academic Medical Center (AMC) in Amsterdam and was carried out in compliance with the Helsinki Declaration. All subjects agreed to participate in the study and written informed consent was obtained from all participants. The trial was registered in the Netherlands Trial Register (NTR3762) before start of the study.

\section{Measurements and analytical procedures}

Serum C-terminal cross-linking telopeptides of collagen type I (CTx), a marker of bone resorption, and serum procollagen type $1 \mathrm{~N}$ propeptide (P1NP), a marker of bone formation, were measured as bone turnover markers. CTx and P1NP concentrations were measured using an immunoassay (Modular Analytics E 170; Roche Diagnostics Corporation, Indianapolis, IN; and Orion Diagnostica, Espoo, Finland, respectively), as described earlier [27]. Plasma normetanephrine was determined by automated online solid phase extraction HPLC-tandem mass spectrometry as described by De Jong et al. [28]. Serum creatinine concentrations were measured on a Hitachi Modular 8000 (C602 and C702) (Roche Diagnostics, Mannheim, Germany). Assays were performed in duplicate and samples from a single subject were assayed within the same analytical batch. Inter-assay coefficients of variation were as follows: CTx $3 \%$, P1NP $8 \%$, and normetanephrine $7.7 \%$.

\section{Effect of clonidine on osteoclast formation and activity}

\section{Cell isolation and culture}

CD $14^{+}$monocytes were isolated from human buffy coats (Sanquin, Amsterdam, Netherlands) obtained from healthy volunteers who provided written informed consent. Briefly, human buffy coats were diluted with phosphate-buffered saline (PBS) containing $1 \%$ citrate $(1: 1)$ and then spun down ( $800 \mathrm{~g}, 30 \mathrm{~min}$, without brake) in Ficoll gradient solution. The interphase containing the peripheral blood mononuclear cells was collected and washed four times in $0.5 \%$ bovine serum albumin buffer ( $2 \mathrm{mM}$ EDTA, phosphate-buffered saline) and then passed through a cell strainer $(40 \mathrm{~mm})$ to ensure the recovery of a clean mononuclear cell population. Cells were counted using a Muse cell counter (Merck Millipore, Darmstadt, Germany) and incubated with CD14 antibody tagged microbeads (Miltenyi Biotec) $\left(1 \times 10^{7}\right.$ cells in $20 \mu \mathrm{L}$ microbead solution) for $15 \mathrm{~min}$ at $4{ }^{\circ} \mathrm{C}$ and then sorted using a manual MACS magnetic cell sorter (Miltenyi Biotec) according to the manufacturer's instructions. This method is frequently used to isolate osteoclast precursors [29]. The purity of the cells was confirmed to be $86 \%$ by flow cytometry (Accuri C6, Becton Dickinson) using a FITC-labeled CD14 antibody and mouse IgG2a isotype antibody (both Miltenyi Biotec) as control. Purified CD14 ${ }^{+}$cells were suspended in culture medium consisting of $\alpha$ MEM (Life Technologies) supplemented with $10 \%$ fetal calf serum (HyClone I), $1 \%$ penicillin/streptomycin (Sigma-Aldrich), and $25 \mathrm{ng} / \mathrm{mL}$ macrophage colony-stimulating factor (M-CSF) (R\&D systems). Bovine cortical bone slices of $650-\mu \mathrm{m}$ thickness were soaked in a 96-well plate with $100 \mu \mathrm{L}$ culture medium for $2 \mathrm{~h}$ prior to seeding. Next, the medium was removed and $75 \mu \mathrm{L}$ of medium containing $25 \mathrm{ng} / \mathrm{mL}$ M-CSF was added to the bone slices, upon which $75 \mu \mathrm{L}$ of cell suspension containing $1.5 * 10^{5}$ cells was added. After 3 days of culturing with M-CSF, the medium composition was changed to $10 \mathrm{ng} / \mathrm{mL}$ M-CSF and $2 \mathrm{ng} / \mathrm{mL}$ receptor activator of nuclear factor kappa-B ligand (RANKL) (R\&D Systems). All cultures were maintained at $37^{\circ} \mathrm{C}$, in a humidified atmosphere under $5 \% \mathrm{CO}_{2}$. Culture media were replaced every 3-4 days.

A time titration experiment was performed first, to determine the appropriate point in time to add clonidine. In this experiment, $\mathrm{CD} 14^{+}$cells were cultured for 28 days, with collection of samples for tartrate-resistant acid phosphatase (TRAcP) staining and bone resorption at days 7, 10, 14, 17, 21,24 , and 28. On day 17, wells were dissolved in RNA lysis buffer from the RNeasy Mini Kit (Qiagen, Hilden, Germany) and stored at $-80{ }^{\circ} \mathrm{C}$ for RNA isolation to determine messenger RNA (mRNA) expression of osteoclast-related genes (tartrate-resistant acid phosphatase (TRAP), cathepsin K (CTSK), calcitonin receptor (CALCR), and dendritic-cell-specific transmembrane protein (DCSTAMP)) and of alpha-2adrenergic receptor subtypes (ADRA2A, ADRA2B, ADRA2C). Based on the bone resorption results from this experiment (data not shown), we decided to add clonidine after 14 days of culturing.

In a separate experiment, clonidine (Sigma-Aldrich) was added to the culture medium after 14 days of culturing in increasing concentrations $\left(10^{-9}, 10^{-7}\right.$, and $\left.10^{-5} \mathrm{M}\right)$. These concentrations were chosen based on peak plasma concentrations achieved after a single oral dose of clonidine $0.3 \mathrm{mg}[30,31]$ and concentrations used in a previous in vitro study using mouse osteoclasts [9]. Wells containing the solvent (water) used for clonidine served as controls. After 14, 21, and 28 days of culturing, wells were washed with PBS and the cells were either fixed in $4 \%$ formaldehyde (used for TRAcP staining) or washed with water (used for bone resorption) and stored at $4{ }^{\circ} \mathrm{C}$. 
All cultures were run at least in fivefold (for TRAcP staining), in tenfold (for bone resorption), and in sixfold (for RNA analysis).

\section{TRAcP staining and cell count}

Osteoclast-like cells were defined as multinucleated (three or more nuclei) tartrate-resistant acid phosphatase (TRAcP)positive cells. After 14, 21, and 28 days of culturing, TRAcP positivity was determined by enzyme histochemistry (Leukocyte Acid Phosphatase Staining Kit, Sigma-Aldrich). Nuclei were stained with diamidino-2-phenylindole dihydrochloride (DAPI). Per bone slice, the number of TRAcP-positive cells with three or more nuclei were counted at a $\times 200$ magnification. We considered multinucleated cells with three to five nuclei as small osteoclasts and multinucleated cells with more than five nuclei as large osteoclasts. The data were expressed as number of $\mathrm{TRAcP}^{+}$multinucleated cells (MNCs) per square centimeter.

\section{Osteoclast resorption assay}

In order to evaluate osteoclast activity, resorption was analyzed after a culture period of 14, 21, and 28 days. Cells were lysed with demineralized water, after which bone slices were sonicated for $30 \mathrm{~min}$ in $10 \%$ ammonium to remove the cell remnants. Bone slices were then thoroughly washed with demineralized water and subsequently incubated in a $10 \%$ saturated alum $\left(\mathrm{KAl}\left(\mathrm{SO}_{4}\right)_{2} \cdot 12 \mathrm{H}_{2} \mathrm{O}\right)$ solution for $10 \mathrm{~min}$. Finally, the bone slices were washed again with demineralized water and the resorption pits were stained with Coomassie brilliant blue. An entire bone slice was micrographed at a $\times 200$ magnification, using a digital camera, mounted on a light microscope (both from Leica, Wetzlar, Germany). We reconstructed the bone slices by merging the micrographs using Adobe Creative Suite Bridge (San Jose, CA, USA). The resorbed area was measured using Image Pro-Plus Software (Media Cybernetics, Silver Spring, MD, USA) and expressed as percentage of the total surface area.

\section{$R N A$ isolation and $q P C R$}

Gene expression of osteoclast-related genes (TRAP, CTSK, CALCR, and DCSTAMP) and of alpha-2-adrenergic receptor subtypes (ADRA2A, ADRA2B, ADRA2C) was quantified by PCR at 17 days of culturing. Total RNA was extracted using the RNeasy Mini Kit (Qiagen) according to the manufacturer's instructions. Reverse transcription of RNA was performed using the MBI Fermentas cDNA synthesis kit (Fermentas, Lithuania), using both the Oligo(dT)18 and the $\mathrm{D}(\mathrm{N}) 6$ primers. Quantitative PCR (qPCR) was performed on the ABI PRISM 7000 Sequence Detection System as described previously [32]. Primers were designed using the
Primer Express software (version 2.0, Applied Biosystems, Foster City, CA, USA) (Table 1). To avoid amplification of genomic DNA, primers were designed such that each amplicon spanned at least one intron. The PCR reactions of the different amplicons had equal efficiencies as determined by serial dilutions of a calibrator sample. For the PCR analysis, porphobilinogen deaminase (PBGD) was used as housekeeping gene. Expression of this gene was not affected by the experimental conditions. Samples were normalized for the expression of PBGD by calculating the $\Delta \mathrm{Ct}\left(\mathrm{Ct}_{\text {gene of interest }}\right.$ $\left.-\mathrm{Ct}_{\mathrm{PBGD}}\right)$. Expression of the different genes is expressed as $2^{-(\Delta \mathrm{Ct})}$

\section{Statistical analysis}

All data were analyzed using SPSS for Windows (version 21.0, SPSS Inc., Chicago, IL, USA). The mean and standard deviation (SD) or the median and interquartile range (IQR) are reported depending on the distribution. To determine the acute effect of a single oral dose of a selective alpha-2adrenoceptor agonist versus no intervention on bone turnover and normetanephrine concentrations, we performed linear mixed model analysis with treatment and time as categorical fixed effects with correction for repeated measurements (AR1). The assumptions of the model were met (e.g., normally distributed residuals and homogeneous variances). For post hoc analysis of bone turnover marker and normetanephrine concentrations at the different time points, we used the paired $t$ test.

Comparisons of the number of TRAcP ${ }^{+}$MNCs and percentage of resorbed bone area over time and between the different culture conditions at day 21 and day 28 were made with a Kruskal-Wallis test, followed by post hoc comparison. All analyses were tested at the 0.05 level of significance.

\section{Results}

\section{In vivo experiment}

\section{Subject characteristics}

We enrolled a total of 12 eligible study participants: four men, and five premenopausal and three postmenopausal women with a median age of 27 (IQR 32) years. All subjects had calcium, albumin, phosphate, $\mathrm{PTH}$, and alkaline phosphatase concentrations within the reference range and normal kidney function. The median $25(\mathrm{OH}) \mathrm{D}$ concentration was 50 (IQR 24) $\mathrm{nmol} / \mathrm{L}$. None of the participants had a (family) history of bone diseases or fractures. Two subjects were current moderate smokers, and two were former smokers. None of the study subjects used $>2$ units alcohol/day. Three premenopausal 
Table 1 qPCR primer sequences

\begin{tabular}{|c|c|c|c|}
\hline Primer & Sequence $5^{\prime} \rightarrow 3^{\prime}$ & $\begin{array}{l}\text { Amplicon } \\
\text { length (bp) }\end{array}$ & Ensembl Gene ID \\
\hline PGBD & TgCAgTTTgAAATCATTgCTATgTC & 84 & ENSG00000113721 \\
\hline TRAP & $\begin{array}{l}\text { AACAggCTTTTCTCTCCAATCTTAgA } \\
\text { CACAATCTgCAgTACCTgCAAgAT }\end{array}$ & 128 & ENSG00000102575 \\
\hline CTSK & $\begin{array}{l}\text { CCCATAgTggAAgCgCAgATA } \\
\text { CCATATgTgggACAggAAgAgAgTT }\end{array}$ & 149 & ENSG00000143387 \\
\hline CALCR & $\begin{array}{l}\text { TgCATCAATggCCACAgAgA } \\
\text { gCATACCAAggAgAAggTCCATAT }\end{array}$ & 78 & ENSG00000004948 \\
\hline DCSTAMP & $\begin{array}{l}\text { ATACTCCAgCCggTgTgTCAT } \\
\text { ATTTTCTCAgTgAgCAAgCAgTTTC }\end{array}$ & 101 & ENSG00000164935 \\
\hline ADRA2A & $\begin{array}{l}\text { AgAATCATggATAATATCTTgAgTTCCTT } \\
\text { gCgAgATCAACgACCAgAAgT }\end{array}$ & 101 & ENSG00000150594 \\
\hline ADRA2B & $\begin{array}{l}\text { TCTggTAgATgCgCACgTAgA } \\
\text { CCCATggCCTCTTCCAgTT }\end{array}$ & 100 & ENSG00000222040 \\
\hline ADRA2C & $\begin{array}{l}\text { gAAggCACggCggAAgT } \\
\text { gTgTggTgCggCgTgTAC }\end{array}$ & 102 & ENSG00000184160 \\
\hline & CCTgCgTCACCgACCAgTA & & \\
\hline
\end{tabular}

For each gene, the first oligonucleotide sequence represents the forward primer and the second sequence, the reverse primer

women used monophasic combined oral contraceptives. All had their tablet-free interval during the control day of the experiment. Two women had a regular menstrual cycle and were in the follicular phase during the intervention with clonidine. The other subjects did not use any medication.

\section{Sympathetic nervous system and bone turnover markers}

Stimulation of alpha-2-adrenoceptors by clonidine leads to a reduction in central sympathetic outflow and a resultant decrease in blood pressure, as well as a decrease in catecholamine release from sympathetic nerves. Plasma normetanephrine, a norepinephrine metabolite, concentrations can be regarded as a marker of sympathetic tone. As expected, blood pressure (data not shown) and normetanephrine concentrations declined after clonidine treatment (Fig. 1a). Baseline CTx (bone resorption) and P1NP (bone formation) concentrations were not different between the intervention and control days $(p=0.398$ and $p=0.196$, respectively). CTx was higher after clonidine compared to the control condition (time*intervention effect, $p=0.035$ ) (Fig. 1b). The effect was most evident $2 \mathrm{~h}$ (11:00) after clonidine administration, with CTx concentrations of $568.5 \pm 150.9$ versus $474.9 \pm 143.1 \mathrm{ng} / \mathrm{L}$ in the control condition $(p=0.001)$. CTx concentrations remained significantly different throughout the remainder of the experiment (13:00, $p=0.005$, and 15:00, $p=0.028)$. P1NP concentrations were not affected by clonidine $(p=0.520)$ (Fig. 1c).

\section{In vitro experiment}

\section{Formation of TRACP $P^{+}$MNCs and bone resorption}

Tartrate-resistant acid phosphatase-positive (TRAcP ${ }^{+}$) multinucleated cells (MNCs) were observed after 14 days of culturing (Fig. 2a). The number of TRAcP $^{+}$MNCs increased in the course of the experiment, with a significant difference in the number of TRAcP $^{+}$MNCs (3-5 nuclei, 5+ nuclei, and total number) between 14 and 28 days of culturing ( $p=0.003$, $p=0.008$, and $p=0.003)$. The total number of TRAcP ${ }^{+}$ MNCs increased from 103 (IQR 32) on day 14 to 649 (IQR 233) $\mathrm{TRACP}^{+} \mathrm{MNCs} / \mathrm{cm}^{2}$ on day 28 of culturing (Fig. 2b).

Bone resorption pits could be visualized at 14 days of culturing (Fig. 3a). In line with the formation of $\mathrm{TRAcP}^{+} \mathrm{MNCs}$, there was an increase in bone resorption from 1.1 (IQR 0.7) \% to 6.9 (IQR 4.6) \% of resorbed bone area between day 14 and day 28 of culturing $(p=0.001)$ (Fig. 3b).

\section{mRNA expression}

At 17 days of culturing, osteoclast-like cells generated from human $\mathrm{CD} 14^{+}$cells expressed mRNA for all investigated osteoclast-related genes, TRAP, CTSK, CALCR, and DCSTAMP (Ct values of 17.5-29.3 cycles), as well as the three alpha-2-adrenergic receptor subtypes ( $\mathrm{Ct}$ values of 26.0-30.1 cycles). 

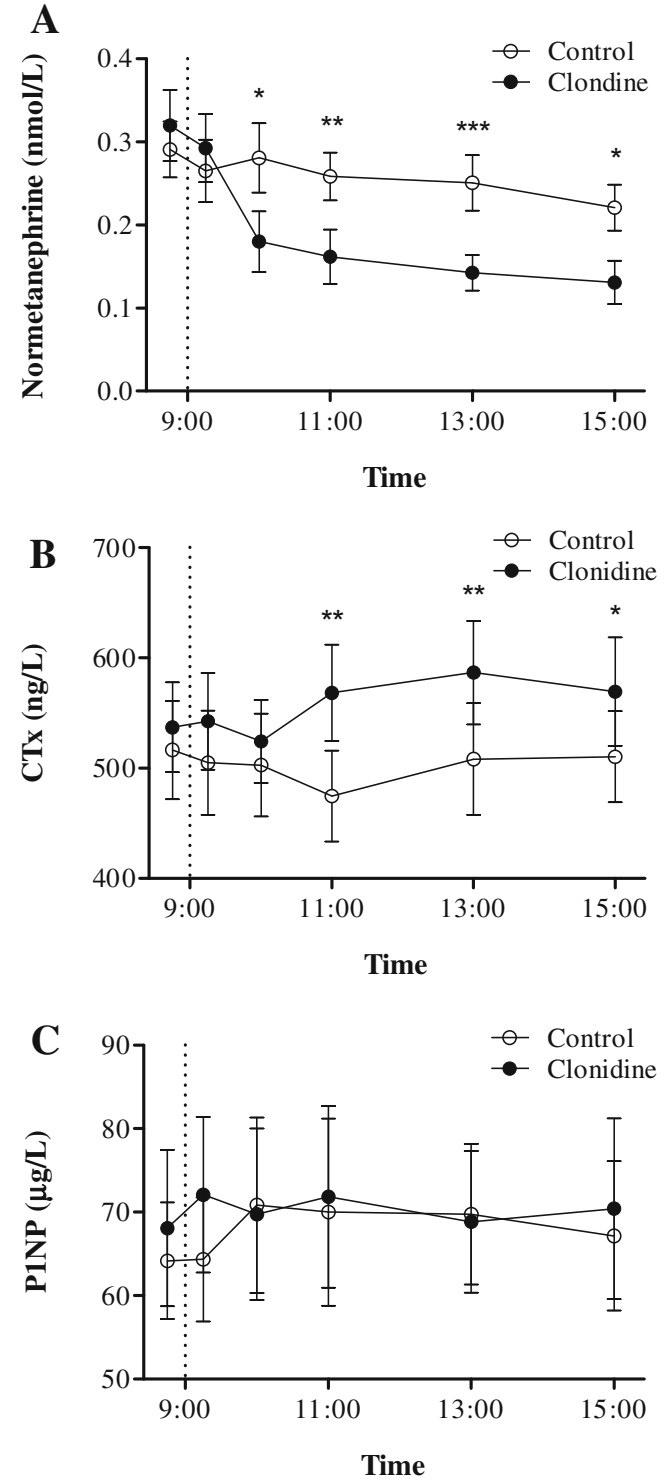

Fig. 1 Normetanephrine (nmol/L) (a); C-terminal cross-linking telopeptides of collagen type I (CTx) (ng/L), a marker for bone resorption (b); and procollagen type $1 \mathrm{~N}$ propeptide (P1NP) $(\mu \mathrm{g} / \mathrm{L})$, a marker for bone formation (c) on the control (open dots) and intervention (closed dots) time points. Normetanephrine concentrations declined after clonidine treatment (time*intervention effect, $p<0.001$ ). CTx was higher after clonidine compared to control (time*intervention effect, $p=0.035$ ). P1NP concentrations were not affected by clonidine (time*intervention effect, $p=0.520)$. Data are expressed as mean \pm SEM. ${ }^{*} p<0.05 ;{ }^{* *} p<0.01$; $* * * p<0.001$

\section{Effect of clonidine on osteoclast formation and osteoclast activity}

No significant effect of clonidine on the number of TRAcP ${ }^{+}$ MNCs (3-5 nuclei, 5+ nuclei, and total number) could be observed after $21(p=0.446, p=0.380$, and $p=0.693)$ and 28 days $(p=0.864, p=0.454$, and $p=0.513$ ) of culturing (Fig. 2b).
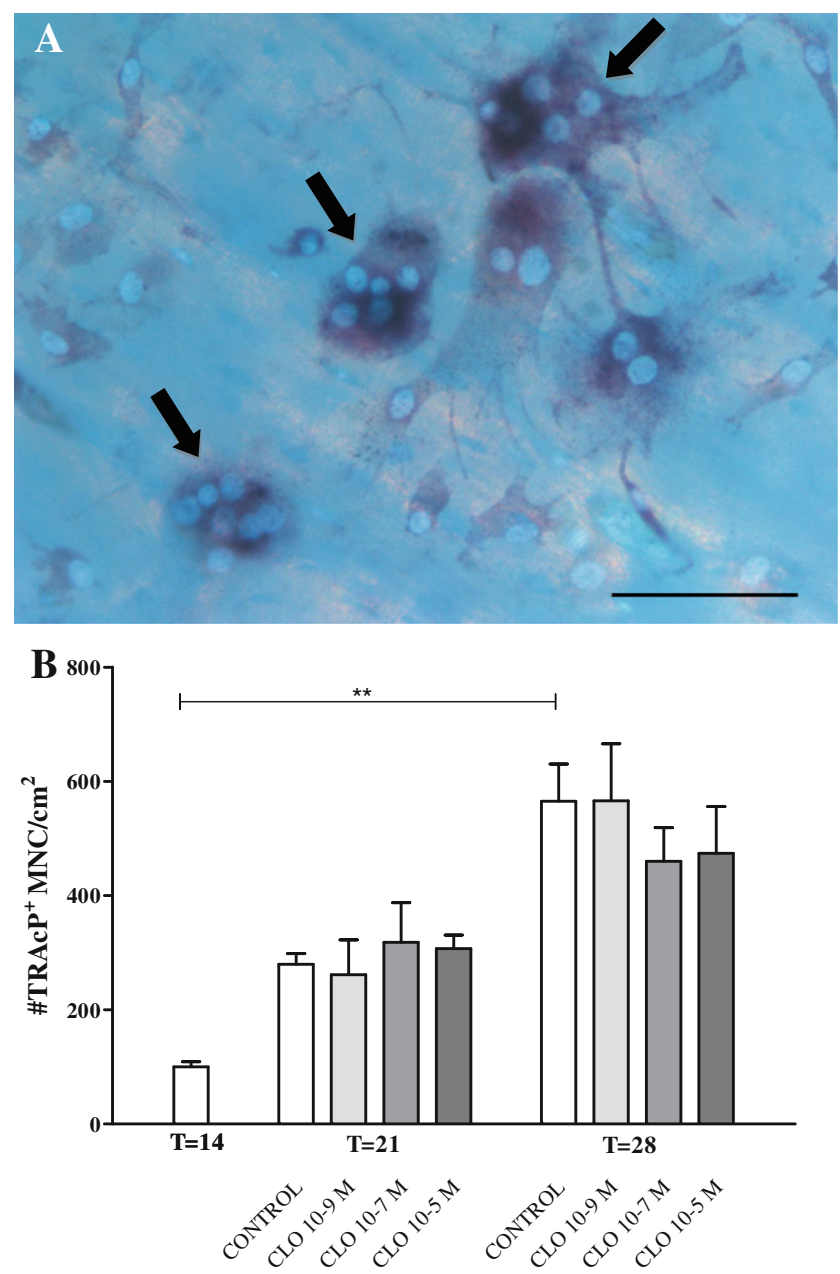

Fig. 2 Representative example of tartrate-resistant acid phosphatasepositive (TRAcP ${ }^{+}$) multinucleated cell (MNC) (three nuclei or more) (arrows) formed from $\mathrm{CD} 14^{+}$monocytes isolated from human buffy coat at day 21. Scale bar $=50 \mu \mathrm{m}$ (a). Formation of TRAcP ${ }^{+} \mathrm{MNCs}$ at culture days 14,21 , and 28. Data are expressed as total number of TRAcP ${ }^{+}$ MNCs per square centimeter (mean $\pm \mathrm{SEM}) .{ }^{* *} p<0.01$. CLO $=$ clonidine (b)

The percentage of resorbed bone area did not also significantly differ between clonidine and control at 21 and 28 days of culturing ( $p=0.789$ and 0.996 , respectively) (Fig. 3b).

\section{Discussion}

In this study, we demonstrated that a pharmacological decrease of the sympathetic nervous system tonus by clonidine, an alpha-2-adrenergic receptor agonist, increases CTx concentrations in humans in vivo, indicating enhanced bone resorption. We demonstrated expression of alpha-2-adrenoceptors by human osteoclast-like cells, but in vitro osteoclast formation and activity were not affected by clonidine.

Our data indicate that pharmacological inhibition of the sympathetic tone increases bone resorption in humans. This 

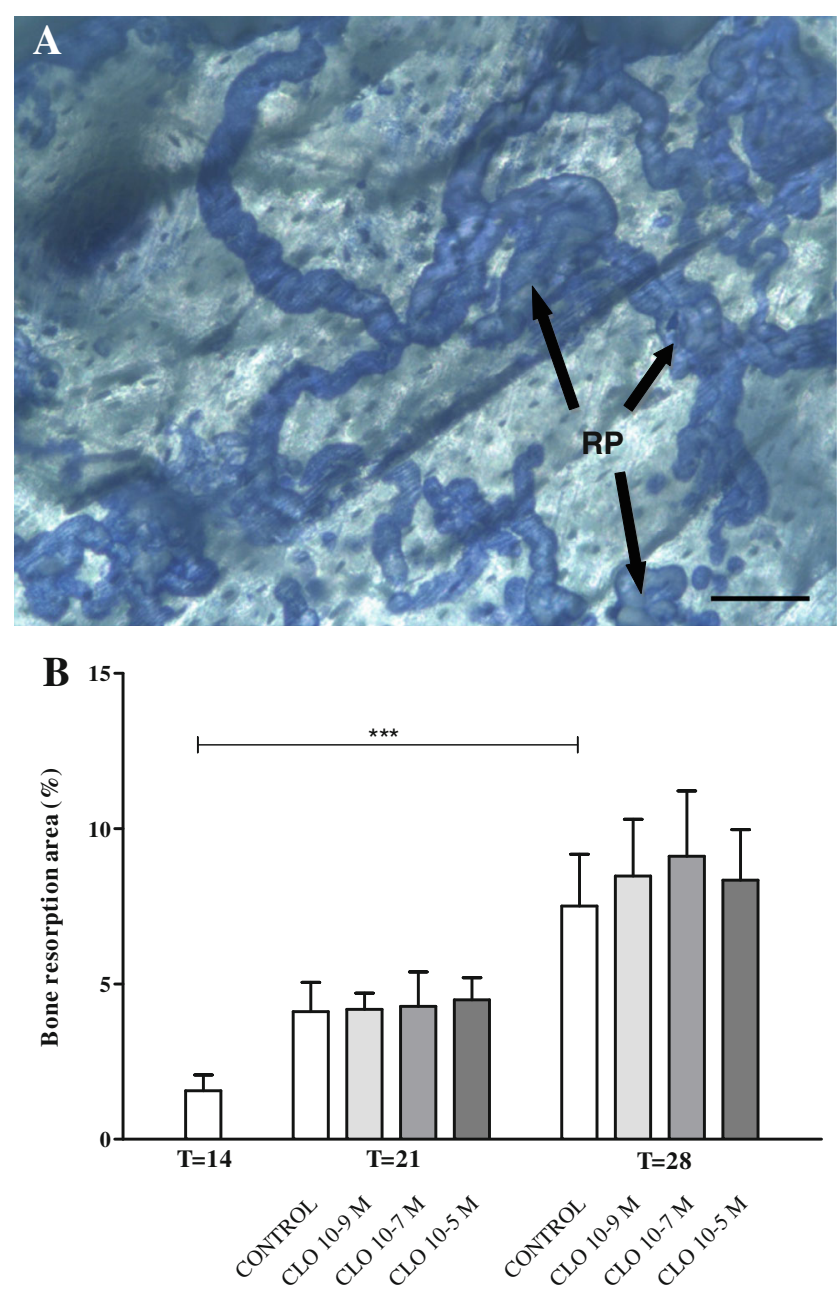

Fig. 3 Representative example of resorption pits (RP) (arrows) stained with Coomassie brilliant blue at day 21 . Scale bar $=100 \mu \mathrm{m}$ (a). Bone resorption at culture days 14, 21, and 28. Data are expressed as resorbed area as percentage of total surface area (mean $\pm \mathrm{SEM}$ ). ${ }^{* * *} p<0.001$. $\mathrm{CLO}=$ clonidine $(\mathbf{b})$

is compatible with recent findings in alpha- $2_{\mathrm{A} / \mathrm{C}}$-adrenergic receptor knockout mice, in which increased sympathetic activity was associated with decreased bone resorption and high bone mass [9]. Both results oppose the general view that the sympathetic nervous system has a catabolic effect on the skeleton $[4,6,5,7,8]$ and suggest that the observed changes in bone resorption are not sympathetically driven but mediated via direct stimulation of the alpha-2-adrenergic receptors on bone cells. To confirm this hypothesis, we investigated the effect of clonidine on osteoclasts that were cultured in vitro. Osteoclast-like cells did express mRNA for all alpha-2adrenergic subtypes, but osteoclast formation and activity were not affected by clonidine. These results are in line with previous observations. Arai et al. showed that treatment with clonidine does not affect the resorbing activity of human osteoclast-like cells generated from bone marrow obtained during artificial hip replacement surgery [33]. In contrast, Fonseca et al. showed that osteoclasts generated from mouse bone marrow increased in number $(51 \%)$ and activity (2.6-fold) in response to clonidine. The clonidine concentrations used were comparable to the concentrations in our experiment [9]. A major difference between this study and ours is that we generated osteoclast-like cells from $\mathrm{CD} 14^{+}$ cells selected from human buffy coats, while Fonseca et al. used osteoclasts that were cultured from mouse long bone marrow. Taken together, these data strongly suggest that osteoclasts generated from $\mathrm{CD} 14^{+}$osteoclasts precursors of human origin do not respond to clonidine whereas those of mouse origin do.

An explanation for the conflicting results from our in vivo and in vitro experiments remains speculative. The clonidine concentrations in the in vitro experiment were based on in vivo peak plasma concentrations achieved after a single oral dose of $0.3 \mathrm{mg}$ clonidine [30,31] and on concentrations from a previous osteoclast culture experiment in rodents [9] and should therefore be sufficient.

The osteoclast-like cells we generated fulfilled the functional criteria of mature osteoclasts, with positive TRAcP staining, formation of resorption pits on bone slices, and expression of osteoclast-related genes. It is, however, not certain that these osteoclast-like cells behave similar to osteoclasts in vivo. In vitro generated osteoclasts are formed by adding cytokines (M-CSF and RANKL) to the culture medium, whereas in vivo the interaction between precursors and cells of the osteoblastic lineage appears to be essential. Therefore, in volunteers, coupling between osteoclasts and other bone cells may have taken place, which is absent in our $\mathrm{CD} 14^{+}$cell cultures. It is therefore likely that clonidine increases bone resorption indirectly, via osteocytes and/or osteoblasts. Indeed, these cells have been reported to express the alpha2-adrenergic receptor [33-36] and osteocytes have recently been recognized as a major player in skeletal activity [37]. This would explain the observed discrepancy between the in vivo and in vitro effects of clonidine.

In vivo $\mathrm{CTx}$ concentrations increased after clonidine administration, indicating increased bone resorption. Clonidine also lowered arterial blood pressure, but not to such an extent that renal perfusion was compromised causing modified CTx clearance. Since plasma creatinine concentrations were not different between the two experimental conditions (data not shown), it is unlikely that the increase in CTx after clonidine is caused by reduced renal clearance of CTx. Other causes for the increase in CTx concentrations also need to be considered. A single oral dose of clonidine increases plasma growth hormone levels [38]. However, in a recent study in obese premenopausal women, growth hormone administration for 6 months did not significantly increase CTx levels [39]. Alpha-2adrenergic receptor agonists have been reported to increase blood glucose levels [40], which might also influence bone turnover. We did not find a significant difference in glucose levels between the clonidine and control day (data not shown), 
making this explanation for the increase in CTx levels unlikely. Although we included in the in vivo experiment a heterogeneous group of only 12 subjects, we could demonstrate a robust effect of clonidine on bone resorption. This strengthens the biological relevance of the effect across different ages and sex. In addition, since baseline bone turnover marker concentrations were not significantly different between the intervention and control day, it is unlikely that other factors account for the changes in CTx concentrations we observed.

The clinical relevance of the increased bone resorption observed with clonidine needs to be considered. Clonidine is licensed for the treatment of hypertension, migraine, and postmenopausal vasomotor symptoms. Of note, postmenopausal women are already challenged with an increase in bone turnover. We did not investigate the long-term effect of clonidine use on bone turnover. However, based on the acute effect of a single oral dose of clonidine, prolonged treatment with clonidine could potentially aggravate the risk of developing osteoporosis. There are currently no studies on the association between long-term use of clonidine and bone mineral density or risk fracture.

In conclusion, an acute decrease in the sympathetic tone by clonidine increases bone resorption in humans in vivo. This effect does not appear to be mediated via direct stimulation of alpha-2-adrenergic receptors on the osteoclast.

\section{Conflicts of interest None.}

Funding Peter H Bisschop is supported by The Netherlands Organization for Health Research and Development (ZonMw) Ref: 90700308 .

Open Access This article is distributed under the terms of the Creative Commons Attribution-NonCommercial 4.0 International License (http:// creativecommons.org/licenses/by-nc/4.0/), which permits any noncommercial use, distribution, and reproduction in any medium, provided you give appropriate credit to the original author(s) and the source, provide a link to the Creative Commons license, and indicate if changes were made.

\section{References}

1. Ducy P, Amling M, Takeda S, Priemel M, Schilling AF, Beil FT, Shen J, Vinson C, Rueger JM, Karsenty G (2000) Leptin inhibits bone formation through a hypothalamic relay: a central control of bone mass. Cell 100(2):197-207

2. Takeda S, Elefteriou F, Levasseur R, Liu X, Zhao L, Parker KL, Armstrong D, Ducy P, Karsenty G (2002) Leptin regulates bone formation via the sympathetic nervous system. Cell 111(3): 305-317

3. Elefteriou F, Ahn JD, Takeda S, Starbuck M, Yang X, Liu X, Kondo $\mathrm{H}$, Richards WG, Bannon TW, Noda M, Clement K, Vaisse C, Karsenty G (2005) Leptin regulation of bone resorption by the sympathetic nervous system and CART. Nature 434(7032):514520. doi: $10.1038 /$ nature 03398
4. Kajimura D, Hinoi E, Ferron M, Kode A, Riley KJ, Zhou B, Guo XE, Karsenty G (2011) Genetic determination of the cellular basis of the sympathetic regulation of bone mass accrual. J Exp Med 208(4):841-851. doi:10.1084/jem.20102608

5. Bonnet N, Laroche N, Vico L, Dolleans E, Benhamou CL, Courteix D (2006) Dose effects of propranolol on cancellous and cortical bone in ovariectomized adult rats. J Pharmacol Exp Ther 318(3): 1118-1127. doi:10.1124/jpet.106.105437

6. Kondo H, Togari A (2011) Continuous treatment with a low-dose beta-agonist reduces bone mass by increasing bone resorption without suppressing bone formation. Calcif Tissue Int 88 (1):23-32. doi: 10.1007/s00223-010-9421-9

7. Bonnet N, Benhamou CL, Brunet-Imbault B, Arlettaz A, Horcajada MN, Richard O, Vico L, Collomp K, Courteix D (2005) Severe bone alterations under beta2 agonist treatments: bone mass, microarchitecture and strength analyses in female rats. Bone 37(5):622-633. doi:10.1016/j.bone.2005.07.012

8. Bliziotes M, McLoughlin S, Gunness M, Fumagalli F, Jones SR, Caron MG (2000) Bone histomorphometric and biomechanical abnormalities in mice homozygous for deletion of the dopamine transporter gene. Bone 26(1):15-19

9. Fonseca TL, Jorgetti V, Costa CC, Capelo LP, Covarrubias AE, Moulatlet AC, Teixeira MB, Hesse E, Morethson P, Beber EH, Freitas FR, Wang CC, Nonaka KO, Oliveira R, Casarini DE, Zorn TM, Brum PC, Gouveia CH (2011) Double disruption of alpha2A- and alpha2C-adrenoceptors results in sympathetic hyperactivity and high-bone-mass phenotype. J Bone Miner Res 26(3): 591-603. doi:10.1002/jbmr.243

10. Rejnmark L, Vestergaard P, Kassem M, Christoffersen BR, Kolthoff N, Brixen K, Mosekilde L (2004) Fracture risk in perimenopausal women treated with beta-blockers. Calcif Tissue Int 75(5):365-372. doi:10.1007/s00223-004-0222-x

11. Rejnmark L, Vestergaard P, Mosekilde L (2006) Treatment with beta-blockers, ACE inhibitors, and calcium-channel blockers is associated with a reduced fracture risk: a nationwide case-control study. J Hypertens 24(3):581-589. doi:10.1097/01.hjh. 0000203845.26690.cb

12. Pasco JA, Henry MJ, Sanders KM, Kotowicz MA, Seeman E, Nicholson GC (2004) Beta-adrenergic blockers reduce the risk of fracture partly by increasing bone mineral density: Geelong Osteoporosis Study. J Bone Miner Res 19(1):19-24. doi:10.1359/ JBMR.0301214

13. Schlienger RG, Kraenzlin ME, Jick SS, Meier CR (2004) Use of beta-blockers and risk of fractures. JAMA 292(11):1326-1332. doi: 10.1001/jama.292.11.1326

14. Levasseur R, Dargent-Molina P, Sabatier JP, Marcelli C, Breart G (2005) Beta-blocker use, bone mineral density, and fracture risk in older women: results from the epidemiologie de l'Osteoporose prospective study. J Am Geriatr Soc 53(3):550-552. doi:10.1111/j. 1532-5415.2005.53178 7.x

15. Reid IR, Gamble GD, Grey AB, Black DM, Ensrud KE, Browner WS, Bauer DC (2005) Beta-blocker use, BMD, and fractures in the study of osteoporotic fractures. J Bone Miner Res 20(4):613-618. doi:10.1359/JBMR.041202

16. Reid IR, Lucas J, Wattie D, Horne A, Bolland M, Gamble GD, Davidson JS, Grey AB (2005) Effects of a beta-blocker on bone turnover in normal postmenopausal women: a randomized controlled trial. J Clin Endocrinol Metab 90(9):5212-5216. doi:10. 1210/jc.2005-0573

17. Bonnet N, Gadois C, McCloskey E, Lemineur G, Lespessailles E, Courteix D, Benhamou CL (2007) Protective effect of beta blockers in postmenopausal women: influence on fractures, bone density, micro and macroarchitecture. Bone 40(5):1209-1216. doi:10. 1016/j.bone.2007.01.006

18. Perez-Castrillon JL, Vega G, Abad L, Sanz A, Mendo M, Porrero MG, Duenas A (2007) Effect of beta-blockers on bone mass and 
biomechanical parameters of the femoral neck in males with acute myocardial infarction. Joint Bone Spine 74(3):259-262. doi:10. 1016/j.jbspin.2006.09.015

19. Meisinger C, Heier M, Lang O, Doring A (2007) Beta-blocker use and risk of fractures in men and women from the general population: the MONICA/KORA Augsburg cohort study. Osteoporos Int 18(9):1189-1195. doi:10.1007/s00198-007-0354-8

20. Yang S, Nguyen ND, Center JR, Eisman JA, Nguyen TV (2011) Association between beta-blocker use and fracture risk: the Dubbo Osteoporosis Epidemiology Study. Bone 48(3):451-455. doi:10. 1016/j.bone.2010.10.170

21. de Vries F, Souverein PC, Cooper C, Leufkens HG, Van Staa TP (2007) Use of beta-blockers and the risk of hip/femur fracture in the United Kingdom and the Netherlands. Calcif Tissue Int 80(2):6975. doi:10.1007/s00223-006-0213-1

22. Veldhuis-Vlug AG, Tanck MW, Limonard EJ, Endert E, Heijboer AC, Lips P, Fliers E, Bisschop PH (2014) The effects of beta-2 adrenergic agonist and antagonist on human bone metabolism: a randomized controlled trial. Bone 71C:196-200. doi:10.1016/j. bone.2014.10.024

23. Toulis KA, Hemming K, Stergianos S, Nirantharakumar K, Bilezikian JP (2013) Beta-adrenergic receptor antagonists and fracture risk: a meta-analysis of selectivity, gender, and site-specific effects. Osteoporos Int. doi:10.1007/s00198-013-2498-z

24. Yang S, Nguyen ND, Eisman JA, Nguyen TV (2012) Association between beta-blockers and fracture risk: a Bayesian meta-analysis. Bone 51(5):969-974. doi:10.1016/j.bone.2012.07.013

25. Veldhuis-Vlug AG, El MM, Endert E, Heijboer AC, Fliers E, Bisschop PH (2012) Bone resorption is increased in pheochromocytoma patients and normalizes following adrenalectomy. J Clin Endocrinol Metab 97(11):E2093-E2097. doi:10.1210/jc.2012-2823

26. Motyl KJ, Rosen CJ (2012) The skeleton and the sympathetic nervous system: it's about time! J Clin Endocrinol Metab 97(11):39083911. doi:10.1210/jc.2012-3205

27. Eekman DA, Bultink IE, Heijboer AC, Dijkmans BA, Lems WF (2011) Bone turnover is adequately suppressed in osteoporotic patients treated with bisphosphonates in daily practice. BMC Musculoskelet Disord 12:167. doi:10.1186/1471-2474-12-167

28. de Jong WH, Graham KS, van der Molen JC, Links TP, Morris MR, Ross HA, de Vries EG, Kema IP (2007) Plasma free metanephrine measurement using automated online solid-phase extraction HPLC tandem mass spectrometry. Clin Chem 53(9):1684-1693. doi:10. 1373/clinchem.2007.087114

29. Davison NL, ten Harkel B, Schoenmaker T, Luo X, Yuan H, Everts V, Barrere-de Groot F, de Bruijn JD (2014) Osteoclast resorption of beta-tricalcium phosphate controlled by surface architecture. Biomaterials 35(26):7441-7451. doi:10.1016/j.biomaterials.2014. 05.048

30. Wing LM, Reid JL, Davies DS, Neill EA, Tippett P, Dollery CT (1977) Pharmacokinetic and concentration-effect relationships of clonidine in essential hypertension. Eur J Clin Pharmacol 12(6): 463-469

31. Keranen A, Nykanen S, Taskinen J (1978) Pharmacokinetics and side-effects of clonidine. Eur J Clin Pharmacol 13(2): 97-101

32. de Vries TJ, Fourkour A, Punt CJ, van de Locht LT, Wobbes T, van den Bosch S, de Rooij MJ, Mensink EJ, Ruiter DJ, van Muijen GN (1999) Reproducibility of detection of tyrosinase and MART-1 transcripts in the peripheral blood of melanoma patients: a quality control study using real-time quantitative RT-PCR. Br J Cancer 80(56):883-891. doi:10.1038/sj.bjc.6690436

33. Arai M, Nagasawa T, Koshihara Y, Yamamoto S, Togari A (2003) Effects of beta-adrenergic agonists on bone-resorbing activity in human osteoclast-like cells. Biochim Biophys Acta 1640(2-3): $137-142$

34. Togari A (2002) Adrenergic regulation of bone metabolism: possible involvement of sympathetic innervation of osteoblastic and osteoclastic cells. Microsc Res Tech 58(2):7784. doi:10.1002/jemt.10121

35. Togari A, Arai M, Kondo A (2005) The role of the sympathetic nervous system in controlling bone metabolism. Expert Opin Ther Targets 9(5):931-940. doi:10.1517/14728222.9.5.931

36. Mlakar V, Jurkovic Mlakar S, Zupan J, Komadina R, Prezelj $\mathrm{J}$, Marc J (2015) ADRA2A is involved in neuro-endocrine regulation of bone resorption. J Cell Mol Med. doi:10.1111/ jcmm. 12505

37. Schaffler MB, Cheung WY, Majeska R, Kennedy O (2014) Osteocytes: master orchestrators of bone. Calcif Tissue Int 94(1): 5-24. doi:10.1007/s00223-013-9790-y

38. Gil-Ad I, Topper E, Laron Z (1979) Oral clonidine as a growth hormone stimulation test. Lancet 2(8154):1242

39. Bredella MA, Gerweck AV, Barber LA, Breggia A, Rosen CJ, Torriani M, Miller KK (2014) Effects of growth hormone administration for 6 months on bone turnover and bone marrow fat in obese premenopausal women. Bone 62:29-35. doi:10.1016/j.bone.2014.01.022

40. Fagerholm V, Haaparanta M, Scheinin M (2011) alpha2adrenoceptor regulation of blood glucose homeostasis. Basic Clin Pharmacol Toxicol 108(6):365-370. doi:10.1111/j.1742-7843. 2011.00699.x 